\title{
Echocardiographic appearance of pulmonary artery stenosis
}

\author{
Jonathan W Swan, John B Chambers, Mark J Monaghan, Graham Jackson
}

\begin{abstract}
Colour flow Doppler mapping can show abnormalities of flow even when the echocardiographic image is equivocal. Routine echocardiography in two patients who were thought to have pulmonary valve stenosis and a ventricular septal defect initially appeared to be normal. Colour flow Doppler mapping, however, showed multiple flow aliasing within the pulmonary artery, approximately $1.5 \mathrm{~cm}$ downstream from the pulmonary valve. More detailed examination by cross sectional echocardiography showed a membrane at this level in both patients. It is possible that more cases of this uncommon anomaly will come to light with increasing use of Doppler techniques.
\end{abstract}

Cross sectional echocardiography provides an anatomical diagnosis in a wide variety of congenital and acquired cardiac lesions. The technique may provide technically poor images in some patients, however, and a morphological abnormality may not be obvious. In these situations, the spatial flow information provided by colour flow Doppler mapping may be diagnostic. ${ }^{1}$ Supravalvar pulmonary artery stenosis is rare, but we have recently used colour flow Doppler mapping to diagnose two cases in which the cross sectional images were equivocal.

\section{Case reports}

CASE 1

A nineteen year old woman in whom valvar pulmonary stenosis had been diagnosed in infancy was seen for outpatient follow up. There was no history of maternal rubella and she had had a normal birth history and developmental milestones. On examination, there was mild bilateral ptosis, but no other neurological or physiognomic abnormality. The supine blood pressure was $120 / 85 \mathrm{~mm} \mathrm{Hg}$ and the pulse rate was 64 beats/minute. The jugular venous pulse was normal and there was no parasternal impulse or abnormality of the apex beat. On auscultation there was a loud ejection systolic murmur, heard best at the left sternal edge in inspiration. The second sound was normal. The electrocardiogram showed sinus rhythm with no evidence of right ventricular hypertrophy and a mean frontal QRS axis of $-40^{\circ}$. The chest $x$ ray was normal.
CASE 2

A thirty one year old man presented with chest pain that was atypical of a cardiac origin. His developmental milestones had been normal, but a ventricular septal defect had been diagnosed in childhood. On examination, the supine blood pressure was $125 / 75 \mathrm{~mm} \mathrm{Hg}$ and the pulse rate was 72 beats/minute. The jugular venous pressure was normal and there was no clinical evidence of right ventricular hypertrophy or left ventricular dilatation. On auscultation there was an ejection systolic murmur which was loudest at the left sternal border, but the second sound was normal. The electrocardiogram and chest $x$ ray were normal.

\section{ECHOCARDIOGRAPHY}

Echocardiographic examination in both cases showed normal right and left ventricular function. There was no right ventricular hypertrophy and no evidence of intracardiac shunts. The cardiac valves were normal in appearance. Initial imaging of the right ventricular outflow tract and pulmonary artery also seemed normal. Colour flow Doppler mapping, however, showed a colour change indicating flow acceleration just beyond the pulmonary valve. This led to a constriction in flow within the main pulmonary artery $1.5 \mathrm{~cm}$ distal to the valve. Beyond this was a turbulent jet directed along the medial border of the artery (fig la). In the second case, there was additional reversed flow through the membrane in early diastole (fig 2a). More detailed examination by cross sectional echocardiography showed a membrane-like stenosis at this point in both cases (figs $1 \mathrm{~b}$ and $2 \mathrm{~b}$ ). The peak velocity in the right ventricular outflow tract and proximal pulmonary artery, measured by pulsed Doppler, was $1.0 \mathrm{~m} / \mathrm{s}$ in both cases. The peak pulmonary artery velocity, recorded by continuous wave Doppler, was $2.8 \mathrm{~m} / \mathrm{s}$ in case 1 and $3.0 \mathrm{~m} / \mathrm{s}$ in case 2 , suggesting peak instantaneous gradients of around $30 \mathrm{~mm} \mathrm{Hg}$. No further investigation is planned in either patient.

\section{Discussion}

Our main purpose is to present the echocardiographic and Doppler ultrasound findings in this uncommon congenital anomaly. There are few reports of the cross sectional echocardiographic appearance of pulmonary artery stenosis and, to our knowledge, none of the Doppler ultrasound findings.

We showed several abnormalities within the pulmonary artery using colour flow Doppler mapping. Acceleration of flow began just 

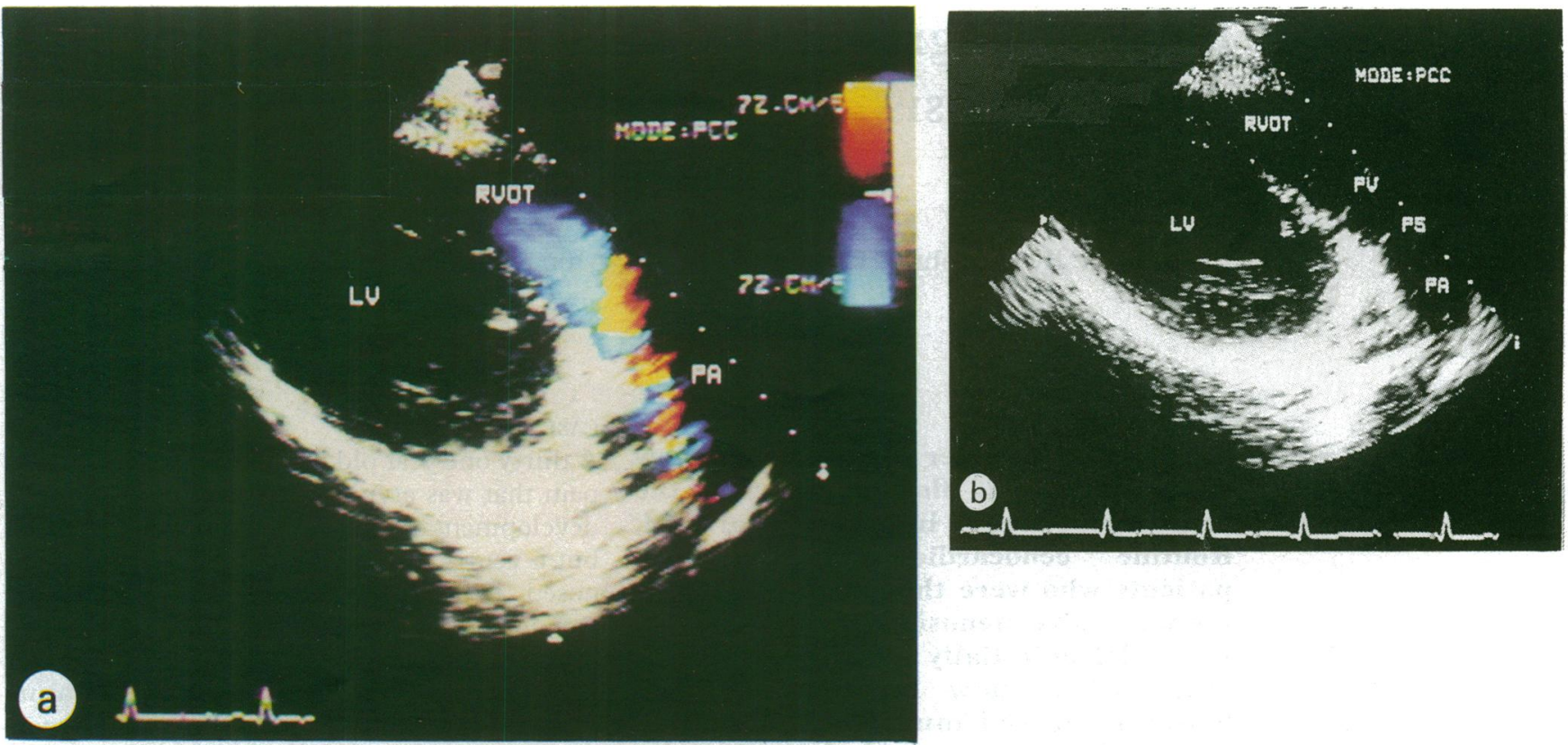

Figure 1 Echocardiographic appearance in case 1 with (a) and without (b) a superimposed colour flow map. A parasternal long axis view was used. The colour system was set at maximum packet size and minimum reject with an enhanced map and spatial filtering. (a) Flow acceleration can be seen immediately downstream from the pulmonary valve leading to a region of flow restriction at a point $1.5 \mathrm{~cm}$ from the valve. Beyond this is a high velocity jet, characterised by multiple mosaic aliasing, directed along the medial border of the pulmonary artery. (b) This shows the underlying anatomy corresponding to these regions of flow disturbance. $L V$, left ventricle; $P A$, pulmonary artery; $P S$, pulmonary artery stenosis; $P V$, pulmonary valve; $R V O T$, right ventricular outflow tract.

downstream from the pulmonary valve and led to a constriction in the cross sectional area available for flow at the point of the stenosis. Downstream from the stenosis was a fast turbulent jet, shown by multiple mosaic aliasing, which was directed along the medial wall of the pulmonary artery. These findings are similar to those reported for aortic coarctation where colour flow mapping may occasionally improve the detection rate. ${ }^{2}$ In the present cases, the stenosis was not immediately obvious on cross sectional imaging and previous examinations had also failed to make the diagnosis. The unequivocal abnormality on colour flow mapping, however, drew attention to the region

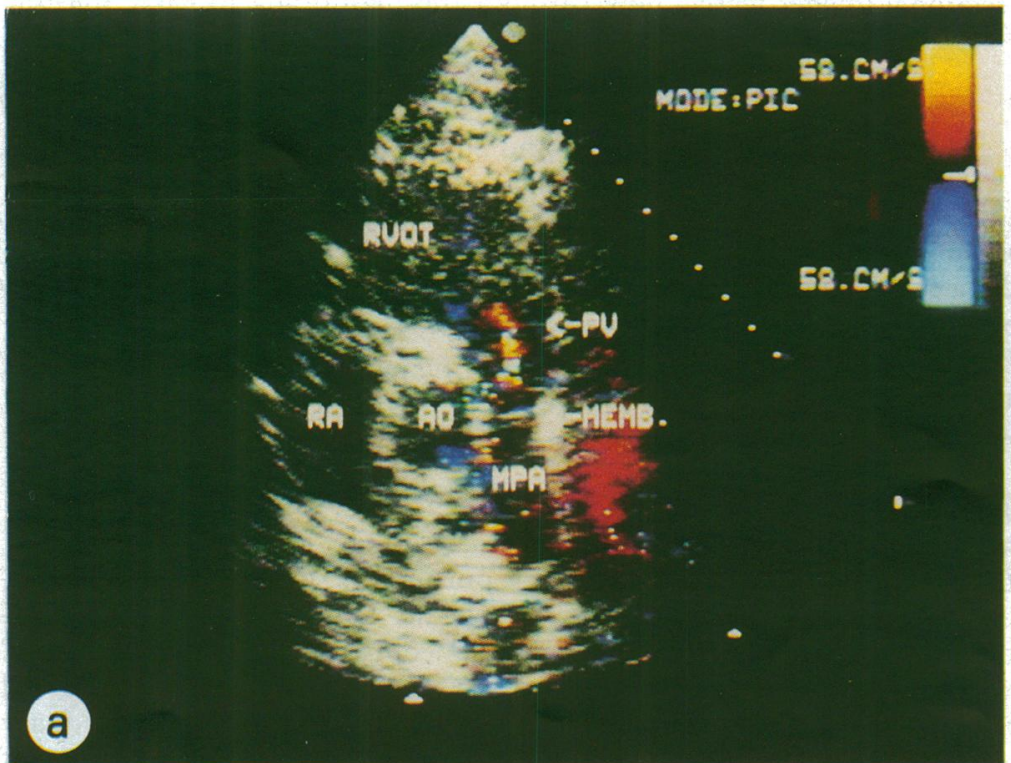

that required further more careful examination by cross sectional echocardiography.

The finding of early diastolic regurgitation across the membrane in one patient is interesting. The pressure drop was greatest at a point just distal to a membranous stenosis, beyond which blood lost momentum with a consequent recovery of static pressure. ${ }^{3}$ We suggest that this pressure gradient beyond the membrane was the reason for transient flow reversal at the start of diastole.

Stenosis of the main or branch pulmonary arteries is uncommon; it is sometimes a result of maternal rubella. ${ }^{4} \mathrm{~A}$ membrane is less common than an elongated stenosis and was seen in

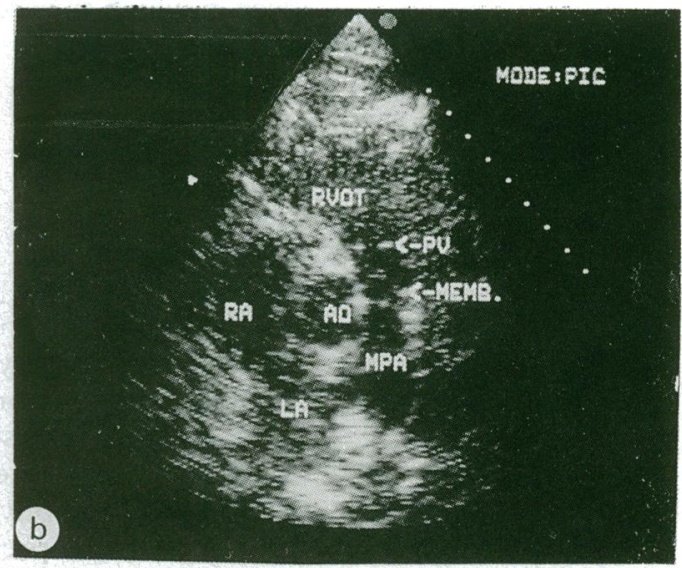

Figure 2 Echocardiographic appearance in case 2 with $(a)$ and without (b) a superimposed colour flow map. (a) An early diastolic frame in a parasternal short axis view. The system was set as in fig la except that spatial filtering was not used. A short, narrow jet can be seen in the main pulmonary artery representing regurgitation of blood past the pulmonary artery membrane. (b) This shows the underlying anatomy. AO, aorta; memb, membrane; $M P A$, main pulmonary artery; $P S$, pulmonary artery stenosis; $P V$, pulmonary valve; $R A$, right atrium; $R V O T$, right ventricular outflow tract. 
two of six patients in Franch and Gay's series in which the main pulmonary artery was affected. ${ }^{5}$ Few long term studies have been undertaken, but it seems that gradients across stenoses in the main pulmonary artery change little over periods of up to 12 years and the prognosis is good in the absence of other associated cardiac anomalies. ${ }^{6}$

Colour flow Doppler is effectively a cross sectional version of pulsed Doppler. Velocity and directional data are gathered at multiple depths down the scan lines of an image sector and then superimposed on the cross sectional image. It therefore screens rapidly for disturbances of flow that may not always be associated with anatomical abnormalities. Furthermore, the detection and localisation of such flow disturbances may be time consuming and of limited accuracy when continuous wave and conventional pulsed Doppler are used. Attention was first directed to the main pulmonary artery in these cases by the unequivocal abnormality on colour flow Doppler mapping. It thus considerably simplified the examination, enabling pulmonary artery stenosis to be diagnosed for the first time in these patients. It is possible that pulmonary artery stenosis is underdiagnosed and that more cases may come to light with the increasing availability and use of Doppler ultrasound techniques. JBC was supported by a British Heart Foundation Junior
Fellowship. The colour flow Doppler system was donated by Mr K Packer.

1 Switzer DF, Nanda NC. Doppler color flow mapping. Ultrasound Med Biol 1985;11:403-16.

2 Simpson IA, Sahn DJ, Valdes-Cruz LM, Chung KJ, Sherman FS, Swensson RE. Colour Doppler flow mapping in patients with coarctation of the aorta: new observations and improved evaluation with color flow diameter and proximal acceleration as predictors of severity. Circulation 1988;77:736-44.

3 Clark C. The fluid mechanics of aortic stenosis-1. Theory and steady flow experiments. J Biomech 1976;9:521-8.

4 Rowe RD. Maternal rubella and pulmonary artery stenosis. Report of 11 cases. Pediatrics 1963;32:180-5.

5 Franch RH, Gay BB. Congenital stenosis of the pulmonary artery branches. A classification with post mortem findartery in two cases. Am J Cardiol 1963;35:512-29.

6 Eldridge WJ, Tinglestead JB, Robertson LW, Mauch HP, McCue C. Observations on the natural history of pulmonary artery coarctation. Circulation 1972;45:404-9.

\section{Addendum}

Since preparing this paper we have demonstrated three further cases of membranous pulmonary artery stenosis, suggesting that it may be more common than previously thought. 